\title{
Los estudios de la(s) masculinidad(es) en la academia universitaria. El caso de México
}

\author{
The masculinity/masculinities studies in University academia. The \\ Mexican case
}

Elva Rivera Gómez y Cirilo Rivera García

\begin{abstract}
Resumen
A más de dos décadas de la expansión de los estudios feministas contemporáneos y, posteriormente, los estudios de la(s) masculinidad(es) es necesario conocer los avances, los obstáculos que han enfrentado estos, así como los nuevos retos en las comunidades científicas mexicanas. Desde el enfoque feminista se presenta un primer esbozo del estudio de los hombres, masculinidad(es) y género y su desarrollo en México.

La disertación contempla los enfoques epistemológicos que han permeado los estudios de las masculinidad(es) desde la década de los noventa a la actualidad; las líneas y resultados de investigación en algunos posgrados del área de ciencias sociales y humanidades en las universidades públicas e instituciones de educación superior. El trabajo forma parte de la línea de investigación Género e Historia del Cuerpo Académico Consolidado de Estudios Históricos de la BUAP e Historia, Género y Educación de la Maestría en Estudios de Género en la Universidad Autónoma de Tlaxcala.
\end{abstract}

Palabras clave: feminismo - estudios de las masculinidades - academia - universidades - México.

\begin{abstract}
It is important to know about the progress and obstacles that contemporary feminist studies have faced after more than two decades and masculinity studies more recently have had. In addition, it is important to know about the chalenges that there are in Mexican scientific comunities. The first draft of the study of men, masculinity (ies), gender and its development in Mexico is presented from the feminist approach.

The lecture will consider the epistemological approaches that have permeated studies of masculinity (ies) from the nineties to the present; topics and research results in some postgrads in social sciences and humanities in public universities and colleges. This task is a part of Gender and History research about the Consolidated Academic Board of Historical Studies at BUAP and History, Gender and Education Master in Gender Studies at Universidad Autonoma de Tlaxcala.
\end{abstract}

Key words: feminism - masculinity studies - academia - universities - Mexico.

Fecha de recepción: Agosto 2015

Fecha de aprobación: Septiembre 2015

Doctora en Historia y Estudios Regionales por la Universidad Veracruzana. Profesora Investigadora del Colegio de Historia de la FFyL de la BUAP. Integrante de la Red Internacional de Estudios de las Masculinidades. E-mail: elva.rivera@gmail.com

2 Maestro en Educación por la BUAP. Profesor de la Maestría en Estudios de Género de la Universidad Autónoma de Tlaxcala. Fundador del Colectivo Construyendo Equidad y del Programa de radio "Varones en la Intimidad". E-mail: ciriga73@gmail.com 


\section{ELFEMINISMOY LOS ESTUDIOS SOBRE LA MASCULINIDAD(ES) EN LAS UNIVERSIDADES}

Como palabras iniciales es importante destacar que la teoría feminista y sus corrientes han contribuido en la construcción del conocimiento crítico al cuestionar el androcentrismo de la ciencia. Asimismo, han criticado las interpretaciones biologicistas, misóginas, sexistas y racistas que han sustentado -y sustentan- la epistemología moderna y contemporánea en las disciplinas e investigaciones.

El feminismo como teoría -y las feministas- ha roto con los paradigmas epistemológicos de la ciencia patriarcal recurriendo a diversas estrategias en las instituciones de educación superior, entre las cuales destacan: a) La legitimación del feminismo como parte del desarrollo del conocimiento científico humanista. b) La formación de especialistas y profesionales por medio de cursos, seminarios, cátedras, diplomados y posgrados. c) La introducción de una agenda de investigación y d) La promoción de vínculos entre la academia y el movimiento amplio de las mujeres -y ahora de los hombres- que retoman al feminismo como una epistemología en sus investigaciones y, además, como parte de la transformación de su vida personal.

En la década de los ochenta del siglo XX en las instituciones de educación superior en México, en especial en las Ciencias Sociales -bajo la influencia de la teoría feminista-, los estudios de la masculinidad inician tímida y aisladamente el estudio de lo que hoy se llama diversidad sexual: la homosexualidad y el movimiento gay.

En el contexto de la crisis del Estado benefactor y el inicio de la economía neoliberal la teoría feminista acuñó el concepto género. Fue entonces que un sector de este transitó de los estudios de la mujer a los estudios de género. Así también los organismos internacionales y el plan de acción de las Conferencias Internacionales de la Mujer ${ }^{3}$ (Beijing, 1995) de Población (Cairo, 1994), entre otras, incorporaron la categoría de género en el diseño y operación de los programas sociales de los gobiernos para coadyuvar a resolver las problemáticas de las mujeres e involucró a los hombres en la atención de políticas sociales, tanto en el espacio privado como público.

En la década de los noventa el feminismo en las universidades mexicanas tuvo sus propios quiebres epistemológicos: permanecer como estudios de la mujer o transformarse en estudios de género. El debate entre las académicas feministas condujo a la disputa académica y teórica para validar la permanencia de los estudios de la mujer. Otras optaron por transformarse en estudios de género. Esta coyuntura posibilitó la creación de Centros y Programas de Estudios de Género. Tal fue el caso de la fundación del Programa Universitario de Estudios de Género de la UNAM y de otros estados de la república mexicana.

En este contexto la introducción de la categoría género visibilizó el estudio de la condición de otra parte de la población, los hombres. A este respecto Cazés ${ }^{4}$ (1998) destaca que antes hubo un antecedente de estudios en el pasado donde sustancialmente se investigó a los hombres,

3 En esta conferencia se incorporaron acciones para involucrar a los hombres en la participación de las políticas de igualdad. En la artículo 107. c) Alentar a los hombres a que participen en condiciones de igualdad en el cuidado de los hijos y el trabajo doméstico y a que aporten la parte que les corresponde de apoyo financiero a sus familias, incluso cuando no vivan con ellas (1985: 44).

$4 \quad$ Murió en diciembre de 2012. Sus aportes fueron muy importantes en la Antropología Social. Acuñó el concepto estudios de género de los hombres. Participó en la fundación de la Academia Mexicana de Estudios de Género de los Hombres (AMEGH). 
la hombría, la virilidad y la masculinidad. Por ello advierte que el inicio de los estudios de los hombres, de masculinidad(es), tienen este antecedente -la década de los noventa- "cuando dio marcha a la intervención de algunos hombres ligados a intelectuales y activistas del feminismo, en la reflexión y la investigación de la condición masculina" (Cazés, 1998: 106).

¿Qué pasa en la academia en relación con la autorreflexión del ser hombre? Es una interrogante que pocos intelectuales sociales se hacen a partir de su praxis académica y política, y más aún cuando algunos de ellos olfatean y coquetean o utilizan mercantilmente estos estudios para convertirse en intelectuales o vacas sagradas sin un compromiso ético, humanista y de transformación personal. Por ello bien vale la pena retomar las palabras de Cazés cuando plantea por qué estudiar(se) como hombre desde el enfoque de género.

"[... a mi juicio se plantea hoy como elemento importante para el desarrollo académico y político de los estudios de género, es la presencia creciente de hombres que buscamos comprender nuestra condición y nuestras situaciones de vida desde la óptica y la experiencia de quienes somos, como género, somos portadores de la opresión [...]" (Cazés, 1998: 107).

Este autor reconoce la herencia epistemológica del feminismo en el estudio del tema de los hombres y de género como académico y activista al afirmar:

\begin{abstract}
"[...] somos académicos y activistas ${ }^{5}$ que actuamos con base en una crítica de la cultura proveniente del marxismo o de los movimientos de las décadas de los sesenta y los setenta, y que hemos comprendido la necesidad de apoyar los movimientos con que las mujeres han ido abriendo los espacios sociales, políticos, culturales, que les han sido vetados durante milenios. Y que hacemos esto con la convicción de que así estamos en el camino de las transformaciones profundas en las relaciones entre los seres humanos, cuyo planteamiento original forma parte del feminismo, del que nos es imprescindible estar cerca para avanzar hacia la convergencia más adecuada, alejándonos de la opresión y de la enajenación" (Cazés, 1998: 107).
\end{abstract}

Es importante conocer desde dónde, qué enfoque y qué metodología se estudia la condición masculina. En la propuesta de Cazés, la metodología de género en los estudios acerca de la condición masculina implica explicar a los hombres en momentos históricos, sociedades y culturas concretas. Y propone los siguientes principios metodológicos para el desarrollo de programas académicos de los estudios de género en los hombres: los aportes de la teoría de género ${ }^{6}$, desigualdades entre hombres y mujeres, desigualdades de los hombres y entre ellos (poderes y privilegios), es decir, en su opinión el estudio de género es un estudio de ejercicio del dominio de género (jerarquización de género, espacios y las formas de opresión de género, de la desigualdad y de los pactos entre los hombres), los pactos patriarcales fundados en la virilidad y fundadores de la violencia, analizar la contribución de los estudios de los hombres y la aplicación de la teoría y la metodología de género por parte de ellos. Por tanto, apunta el autor, es pertinente ante la urgencia de deconstruir concepciones y actitudes masculinas patriarcales, tanto en la vida pública como en la intimidad de la privada [...] (Cazés, 1998, pp. 113-114). En este sentido es importante señalar que en la academia universitaria mexicana está pendiente un estudio en torno a las premisas teóricas

5 En el caso de los académicos que impulsaron estos estudios en las universidades mexicanas, la mayoría provino de la clase media urbana.

6 Seguimos la propuesta de Scott en el señalamiento que hace en torno al empleo de la categoría género. Hay autores como Cazés que denominan la teoría de género. Propuesta que es discutida al interior de la teoría feminista contemporánea. 
y metodológicas que sustentan las investigaciones sobre los estudios de las masculinidades en los estudios de posgrado ${ }^{7}$.

El eje central de análisis del estudio de la masculinidad, sostienen Gloria Careaga y Salvador Cruz, es el poder, concepto fundamental en el estudio de género y, particularmente en el campo de la masculinidad (Careaga y Cruz, 2006: 11).

Al finalizar el siglo XX y principios del siglo XXI los estudios de los hombres, los varones y las masculinidades se posicionan en el discurso político, académico y -sobre todo el último- en las políticas públicas ${ }^{8}$. En las universidades se inició la reflexión de este tópico en los seminarios, cursos y líneas de investigación ${ }^{9}$.

El Programa Universitario de Estudios de Género (PUEG) de la UNAM desde 1993 abrió una línea de investigación. El "Proyecto de estudios de la masculinidad y sus implicaciones sociales" en el que participó Daniel Cazés. A partir de esto se derivaron algunas publicaciones. Un trabajo pionero fue "La dimensión social del género: posibilidades de vida para mujeres y hombres en el patriarcado", en Antología de la Sexualidad Humana (1994).

Así también el PUEG desarrolló cuatro seminarios donde además no solo fue un espacio de reflexión entre integrantes de la academia de diversas instituciones sino también de vinculación con las ONG y las instancias gubernamentales. Las temáticas estudiadas fueron las siguientes: El ejercicio del poder y la masculinidad; Modelos de desarrollo y masculinidad; Políticas públicas y masculinidad; Masculinidad y participación y Acción ciudadano. Este ejercicio interinstitucional fue muy importante, refieren Careaga y Cruz [...] no solo de reflexión teórica y de incidencia en los proyectos de intervención social y en el diseño de las políticas públicas (Careaga y Cruz, 2006: 15).

El apoyo del PUEG de la UNAM en la institucionalización de los estudios de género fue vital en las universidades del interior de la república mexicana. Este fue el caso del Centro de Estudios de Género de la Benemérita Universidad Autónoma de Puebla, pues en 1998 se realizó el Primer Seminario "Masculinidad y Cultura" (Rivera, 2006), donde confluyó la academia, el sector gubernamental y la sociedad civil como un ejercicio de aprendizaje colectivo e interinstitucional a nivel estatal.

Se requiere de una revisión más profunda para analizar las propuestas metodológicas que plantea Cazés, desde el uso de los conceptos y metodología que se han privilegiado hasta ahora en cada disciplina e institución académica.

$8 \quad$ A partir de la CIPD (1994) en el Cairo, se impulsaron acciones de reflexión en la agenda pública acerca de la importancia de incorporar a los hombres para la construcción de la igualdad, un ejemplo de ello fueron: en la Comisión de Naciones Unidas sobre el Estatus de las Mujeres en su documento: "El papel de hombres y niños en el logro de la igualdad de Género". En México, a partir del 2012, la Ley Federal del Trabajo establece la Licencia de Paternidad en el artículo 132, fracción XXVII Bis. Que dice: "Otorgar permiso de paternidad de cinco días laborables con goce de sueldo, a los hombres trabajadores, por el nacimiento de sus hijos y de igual manera en el caso de la adopción de un infante".

9 Derivado de la formación en los estudios feministas en el Taller de Antropología de la Mujer del Colegio de Antropología Social de la Universidad Autónoma de Puebla impartido por Marcela Lagarde y más tarde dirigido por María Eugenia D’Aubeterre y Patricia Castañeda Salgado, este se transformó en Seminario de Estudios de Género. Así, en 1996, la primera tesis que abordó el tema de las masculinidades desde el enfoque feminista fue A todos los que quieren y aman el juego del hombre: el juego, una forma de expresión de la masculinidad de los obreros de la Volkswagen, de Fernando Huerta Rojas (Rivera, 2010). 
Más tarde, en el PUEG este programa continuó con la reflexión teórica en esta temática. En el 2000 Javier Alatorre coordinó el área de Masculinidad. Los objetivos fueron, entre otros: estimular la investigación, la capacitación y acción en masculinidad con el propósito de lograr una sociedad más equitativa entre los sexos. Alatorre señaló que en el seminario principalmente:

1. [...] revisamos los distintos aportes teóricos en esta área y las principales tensiones que tienen que desarrollarse para poder entender los procesos de construcción y reproducción de la masculinidad. [...] han participado profesores nacionales e internacionales como Teresita de Barbieri, Matthew Gutmann, Ondina Fachel y Victor Seidler. Al Seminario asisten investigadores(as), representantes de instituciones gubernamentales y no gubernamentales, esperamos apoyar las labores de investigación, enseñanza y desarrollo de acciones y políticas públicas en México sobre la masculinidad.

\section{Investigación: estamos realizando investigación sobre sexualidad masculina y paternidad.}

3. Capacitación: estamos desarrollando un modelo de capacitación para prestadores de servicio del sector salud para incorporar a los hombres a las acciones en salud sexual y reproductiva (Alatorre, 2000).

A pesar de estos avances en este campo de conocimiento, en la academia universitaria mexicana aún prevalece la exclusión, las resistencias, la misoginia, el androcentrismo y se cuestiona al feminismo por su crítica al pensamiento racional patriarcal en las instituciones de educación superior ${ }^{10}$. Al respecto coincidimos con Núñez (2004) cuando advierte que los estudios de las masculinidades vive un proceso similar al de los estudios feministas, pues "los hombres" sujetos genéricos son excluidos del conocimiento de diferentes formas:

\section{1. [... ] en los cuerpos tradicionales del conocimiento, sobre todo cuando los comportamien- tos resultantes de una socialización de género se consideran producto de su "naturaleza" o cuando se les trata como seres universales.}

2. [...] como poseedores de conocimiento, cuando se les priva de la posibilidad de conocer de otras maneras, conocer por medio de sus emociones, de sus dinámicas deseantes, de sus experiencias corporales, en la medida que se les conmina a actuar racionalmente y a despreciar o reprimir las características simbólicamente "femeninas".

3. [...] en la medida en que no se conozcan a sí mismos en tanto sujetos genéricos, y no sean capaces de encontrar una relación entre sus malestares, sus "problemas sin nombre", y su construcción genérica.

4. [...] en la medida en que se les prive de credibilidad como seres capaces de producir conocimiento ("objetivo") por considerárselas incapaces de "objetividad" en la medida en que son considerados simbólicamente femeninos [...]

5. [...] por la concepción misma de lo que cuenta como conocimiento y por las concepciones sobre la razón y la objetividad. Las experiencias de los propios "hombres" y sus saberes sobre su experiencia como "hombres", es decir, como sujetos genéricos, o sobre su socialización

10 Consuelo Miqueo, Ma. José Barral y Carmen Magallón (2008), reúnen trabajos académicos de investigaciones de diversos países, que presentan resultados de orden cuantitativo y cualitativo que develan las inequidades en el campo científico, además se señalan las diferentes formas de exclusión de las mujeres investigadoras a partir del modelo androcentrismo científico. 
como sujetos genéricos, muchas veces se excluyen porque se les considera "subjetivas", así como "no masculinas" (Núñez, 2004: 29-30).

La exclusión de los saberes de las mujeres y los hombres como sujetos genéricos ha obligado a un sector del feminismo, y a los hombres que se adhieren a esta corriente, a reflexionar respecto de la masculinidad, es decir, la manera en cómo se configuran y reconfiguran las identidades genéricas a partir de las transformaciones estructurales, económicas, sociales y culturales que generan cambios en las relaciones de género. De tal forma que en México tenemos desde hace unos años obras colectivas y foros académicos donde se han analizado los aspectos teórico-metodológicos de estos.

Como resultado del debate epistemológico de la masculinidad, Amuchástegui propone, en términos metodológicos, el empleo del concepto "Construcción de la masculinidad" para:

[...] designar una serie de discursos y prácticas sociales que pretenden definir al término masculino del género dentro de configuraciones históricas particulares, diferenciándolo de las propias experiencias de los hombres, que no están reducidos a someterse a tal construcción y que manifiestan innumerables formas de resistencia (Amuchástegui, 2006: 175).

Es importante señalar que tanto los estudios feministas como los estudios de la masculinidad no son "aceptados"11 por la academia tradicional, por eso hay disputas en el campo de las ciencias sociales y resistencias para reconocer sus aportes. A pesar de la reorganización -con tintes académicos reformistas- que se ha impuesto en el sistema de educación superior en México, estos estudios han tenido que adecuarse a las políticas financieras en la gran mayoría de las instituciones en el país.

Por ejemplo, el Programa Interdisciplinario de Estudios de la Mujer del Colegio de México (PIEM-COLMEX) tuvo que sortear una reestructuración y reubicarse en el Centro de Estudios Sociológicos, a pesar de tener una trayectoria muy importante en la formación de los estudios de las mujeres, este no pudo mantener su autonomía académica. A pesar de esta situación, es importante apuntar que este espacio académico formó a especialistas en los cursos de verano y de especialización, y además ha contribuido a la expansión de estos estudios en otros centros universitarios. Un aporte al estudio de las masculinidades fueron los trabajos pioneros de Ana Amuschástegui e Ivonne Szasz (2007), y recientemente este programa fundó la Revista Interdisciplinaria de Estudios de Género (2015).

Por otra parte, en la Universidad de Guadalajara, Juan Carlos Ramírez ${ }^{12}$ (2008) condujo los trabajos del Segundo Congreso sobre varones y masculinidades en el 2006, donde además fue la sede y fundación de la Asociación Mexicana de Estudios del Género de los Hombres (AMEGH). Él es pionero en los estudios de la violencia y las masculinidades. Una obra colectiva coordinada con Griselda Uribe Vázquez fue publicada en 2008.

11 Nos hemos encontrado casos de algunas/os estudiantes de posgrado que presentan sus proyectos de investigación desde las teorías feministas o de género como categoría de análisis, son menospreciados por los comités académicos o simplemente ignoran sus proyectos.

12 Sus primeros trabajos se dedicaron al tema de la salud sexual y reproductiva, posteriormente incursionó a los estudios de las masculinidades. En la página de la UdeG, el Centro de Investigación no registra las publicaciones del autor. Por tanto, esta es una forma de invisibilizar sus resultados de investigación, tanto al interior como al exterior de la comunidad académica. 
Reconocer las investigaciones de las feministas en la academia no ha sido fácil. Lo mismo enfrentan los estudios de las masculinidades. Por ejemplo rastrear en los portales institucionales ${ }^{13}$ los temas de tesis y sus respectivas direcciones en los posgrados de los pioneros en los estudios de la masculinidad ha sido difícil. Suponemos que prevalece en estas los sistemas clásicos del resguardo físico y la digitalización implica tal vez riesgos como el plagio. Esta situación impidió indagar ampliamente las investigaciones en la gran mayoría de posgrados del área de ciencias sociales y humanidades, en especial sobre los y las tesistas de alguno de estos académicos y académicas ${ }^{14}$.

Guillermo Núñez Noriega, del Centro de Investigación en Alimentación y Desarrollo, A. C., en Sonora, desarrolla la Línea de Género y Grupos Vulnerables desde el 1 de septiembre de 1997. A pesar de su experiencia y aportes en el campo de las masculinidades, la línea no aparece registrada oficialmente ${ }^{15}$. En la misma situación se encuentra Fernando Huerta adscrito al Centro de Estudios Interdisciplinarios de Género y a la academia de Arte y Patrimonio Cultural de la Universidad Autónoma de la Ciudad de México. Uno más es el de Rodrigo Parrini Roses de la Universidad Autónoma Metropolitana-Xochimilco. En su caso, se da cuenta que en el 2011 impartió el Seminario Teorías y metodologías para el estudio de las masculinidades en la Universidad Autónoma de San Luis Potosí.

Si de reflexión y crítica académica se trata es vital subrayar que los congresos, coloquios y encuentros son un espacio de reflexión de los estudios relativos a la masculinidad, los varones, y los hombres. El primero de ellos fue organizado por Guitté Hartog y Elva Rivera en la Benemérita Universidad Autónoma de Puebla en el 2004, donde se fundó la Red Internacional de Estudios sobre varones y masculinidades y, más tarde, se inició la publicación digital de la Revista La Manzana (Rivera, 2006).

La Asociación Mexicana de Estudios del Género de los Hombres (AMEGH) fue impulsada por un grupo de académicos e integrantes de la sociedad civil que decidieron en el 2005 institucionalizarla como Asociación Civil. El primero en presidirlo fue Guillermo Núñez Noriega. Esta asociación ha construido un repositorio digital que alberga tesis de posgrado y de grado, artículos y ensayos acerca de tópicos dedicados a los estudios de género de los hombres. Sin embargo para tener acceso a esta base de datos y material se requiere de un pago simbólico ${ }^{16}$.

13 En las dos últimas décadas en México, gracias a la Ley de Transparencia, se ha transitado a la digitalización de la información, la que ha coadyuvado a dar a conocer parte de la documentación oficial y consultar sus acervos virtuales. El Consejo Nacional de Ciencia y Tecnología (CONACYT), por medio del Programa Nacional de Posgrados de Calidad (PNPC), ha impulsado la digitalización de la información, entre estas se ubican las tesis. Parcialmente solo algunos posgrados y centros de investigación han ubicado en sus portales virtuales una sección de tesis. Por lo que para complementar la información se requiere de la consulta física de cada una de las bibliotecas para ampliar la información respecto de este tema.

14 Por ello es importante contar con un portal digital que albergue de manera gratuita el repositorio de tesis de grado y posgrado. En cambio en la redes de los colectivos que trabajan la temática desde la sociedad civil sí aparecen las líneas de acción y entrelazados entre redes colectivas.

15 Para algunos cuerpos académicos - grupos de investigación reconocidos al interior de las instituciones educativas en México-, los estudios de las mujeres, género y de las masculinidades tienen poca relevancia; los aportes a la ciencia social y de las humanidades aún no es reconocida como área de conocimiento en la evaluación del Sistema Nacional de Investigadores del Consejo Nacional de Ciencia y Tecnología (CONACYT). De tal manera que es necesario investigar a profundidad si son o no reconocidos y visibles estas investigaciones en las universidades y centros de investigación.

16 Es importante señalar que la mayoría de las investigaciones de grado y posgrado han sido desarrolladas con recursos públicos federales o estatales. Por tanto, el acceso a estos debería ser libre. En el contexto del neoliberalismo las Asociaciones Civiles u Organizaciones de la Sociedad Civil se han visto obligadas a autofinanciarse. Esto también 
Por tanto, sus congresos (2006-2013) han sido un espacio de vinculación entre la academia y la sociedad civil. Rivera (2014) recomienda realizar investigaciones a futuro para conocer la incidencia de los estudios de género y de las masculinidades y los resultados obtenidos en el binomio academia-sociedad civil, ante la ausencia de una formación feminista de quienes se dedican a la capacitación en temas de la perspectiva de género y masculinidades financiados por las instituciones públicas.

A esta iniciativa se suman dos trabajos que abordan el análisis de los hombres activistas con el feminismo. El primero de ellos es un trabajo colectivo coordinado por Tania Escalante e Ignacio Lozano (2014), dicho trabajo aborda reflexiones teóricas, experiencias de trabajos con hombres desde diferentes aristas: sexualidad, violencia, homofobia, entre otros. El segundo, David Pinilla (2014) analiza en su investigación las formas en que los hombres participan en el activismo en grupos para detener la violencia masculina, igualdad de género y en la diversidad sexual.

Por otra parte, la mayoría de los académicos universitarios, en particular los científicos sociales, hasta ahora no han incorporado en sus investigaciones a los hombres como sujetos genéricos. De tal forma que estos no forman parte del corpus teórico metodológico de sus investigaciones. Por ello, Núñez reconoce, que:

\section{[...] entre los "hombres" existen saberes subyugados sobre sí mismos como sujetos genéri- cos. Las historias y saberes sobre su subjetividad, su identidad y sus prácticas suelen quedar excluidos del conocimiento, sobre todo si implican saberes que son objeto de represión y negación social que no caben en las definiciones dominantes de "conocimiento" ni de ser "hombres" (Núñez, 2004: 30).}

En los primeros años del siglo XXI la creación de las Maestrías en Estudios de Género del Colegio de México (2010) y la Maestría en Estudios de la Mujer de la Universidad Autónoma Metropolitana-Xochimilco han sido espacios académicos de formación en la investigación feminista y en los estudios sobre la masculinidad(es). Además han contribuido a generar nuevas líneas de investigación en otros posgrados de ciencias sociales y humanidades, cuyos cursos, seminarios y dirección de tesis son un aporte más de las académicas feministas y de académicos que se adhieren a este campo epistémico.

\section{LOS ESTUDIOS DE LAS MASCULINIDADES EN LOS POSGRADOS EN MÉXICO, UN PRIMER ACERCAMIENTO}

El desarrollo de los estudios feministas a los largo de las dos últimas décadas del siglo XX posibilitó que en el nuevo milenio en algunas universidades se diera paso a la creación de líneas de investigación acerca de estudios de género y masculinidad, y además su institucionalización ha generado el desarrollo de investigaciones en los estudios de posgrados ${ }^{17}$.

ha forzado a buscar recursos alternos mediante cuotas "simbólicas". De tal forma, deberíamos reflexionar hasta dónde estas políticas públicas han orillado a mercantilizar no solo los estudios feministas, de género y los estudios de las masculinidades. Luego entonces habría que preguntarse qué hacer para compaginar el binomio academia del sector público-sociedad civil.

17 A continuación se presenta la primera recopilación de tesis de posgrado desarrolladas en instituciones de educación superior en México. Resta mencionar que está pendiente un análisis acerca de los enfoques y metodologías en estas investigaciones. 
En el 2001 Nelson Minello del Centro de Estudios Sociológicos del COLMEX obtuvo el grado de Doctor en Ciencias Sociales con especialidad en Antropología Social por el Centro de Investigaciones y Estudios Superiores en Antropología Social (CIESAS-Occidente), con la tesis La masculinidad en México al fin del milenio.

En el Colegio de México las disertaciones doctorales en Ciencias Sociales del Centro de Estudios Sociológicos en el tema que nos ocupa fueron las siguientes: Calvario, José Eduardo (2014). Género y masculinidad, juegos de poder y configuración del peligro en el poblado Miguel Alemán, Sonora, y la de Lara Carmona, Vanessa Lizbeth (2012). Masculinidades en el trabajo. Lógicas de acción y definiciones subjetivas de varones enfermos. Ambas fueron asesoradas por Nelson Minelo.

En el Doctorado en Estudios de Población de esta misma institución se localizaron las tesis de Gallego Montes, Gabriel (2007). Patrones de iniciación sexual y trayectorias de emparejamiento entre varones en la ciudad de México. Una mirada biográfica-interaccional en el estudio de la sexualidad, asesorada por la Dra. María del Rosario de Fátima Juárez Carcaño; y la de Rosas Mujica, Carolina Alejandra (2006). Varones al son de la migración. El papel de la migración internacional en la configuración de la/s masculinidad/es: Estudio cualitativo en una localidad veracruzana y en Chicago, bajo la dirección del Mtro. Manuel Ángel Castillo García.

En la Universidad Veracruzana se han sustentado las tesis doctorales de De Keijzer Fokker, Benno George Álvaro (2010). Masculinidades, violencia, resistencia y cambio, en el Doctorado en Salud Mental Comunitaria, y como director de tesis el Dr. Roberto Castro Pérez, del CRIM de la UNAM. Así también en el Doctorado en Historia y Estudios Regionales de esta misma universidad se presentaron las siguientes disertaciones: Cancino Barffusón, Sergio René (2012). Permanencias, cambios y tensiones en el proceso de visibilización de la homosexualidad masculina en Xalapa, Veracruz 1969-2000; Guzmán Ibáñez, María de Lourdes (2009). Violencia conyugal y dominación masculina. Veracruz (1990-2005), y Belmonte Juárez, Gregorio (2007). Construcción de masculinidades e incidencia en el embarazo en adolescentes del Valle del Yaqui 2000-2004. Todas bajo la dirección de la Dra. Rosío Córdova Plaza.

En el Colegio de Michoacán, en el Centro de Estudios de Antropología, se presentó la tesis doctoral de Oscar Misael Hernández Hernández (2007). La producción de hombres ordinarios. Procesos históricos y construcción de las masculinidades en Tamaulipas. Además, es importante reconocer la contribución de Misael (2008) al campo de estudios que nos ocupa el balance historiográfico en las investigaciones de las y los especialistas internacionales y nacionales en torno a los aspectos teórico-metodológicos empleados en los estudios de las masculinidades en México.

La Maestría en Estudios de Género del Centro de Estudios Sociológicos del COLMEX, desde su creación en el 2005 a la fecha ha tenido cinco generaciones y se han sustentado 61 tesis: 10 (2012-204); 13 (2012-2010); 11 (2009-2007); 16 (2007-2005); 11 (2005-2003). En estas disertaciones prevalecen los estudios sobre las mujeres. Son escasas las dedicadas a los estudios de las masculinidades y hombres. Entre estos se ubican las siguientes: García Reyes, Fidel (2014). Visibilizar a los invisibles: representación y militancia en la fotografía y pintura expuesta en los primeros 25 años de la Semana Cultural Lésbico-Gay en el Museo Universitario del Chopo, bajo la asesoría deAna María Tepichin y la codirección del Dr. Rodrigo Laguarda; Giraldo Aguirre, Sebastián (2014). Padres en suspenso. Proceso de decisión, significados y prácticas de paternidad de algunos varones gay en la Ciudad de México; González Romero, Martín 
Humberto (2014). Hombres de la nación. Masculinidad y modernidad en tres novelas del México independiente, 1857-1869; Amezcua Juárez, Pascal Dominique (2012). Experiencias de género en las narrativas autobiográficas de Gioconda Belli y Sergio Ramírez. Un estudio sobre masculinidades y feminidades dentro del sandinismo; Viveros Pérez, Francisco Eduardo (2012). Masculinidad y cáncer. La (re)significación de la(s) masculinidad(es) en las experiencias del cáncer genital. Las dos últimas asesoradas por Nelson Minelo.

Entre el 2005 y 2009 se identificaron los siguientes trabajos: Mena Méndez, Paulina (2009). Ser padres solteros en la Ciudad de México el Área Metropolitana; Montoya Gómez, María Victoria (2009). Amancebamientos y concubinatos. El discurso normativo y las representaciones de lo femenino y lo masculino. La Ciudad de México y sus alrededores, 1777-1805, cuya asesora fue Pilar Gonzalbo; Flores Zúñiga, Claudia Ruth (2007). Algunos elementos del ejercicio de la masculinidad en un grupo de sacerdotes de la Iglesia católica; Narváez Lozano, Alfredo (2007). Ciudadanía y orientación sexual: cambio social en la inclusión de la homosexualidad en el México de inicios del siglo XXI. Asesorada por María Soledad Loaeza Tovar; Rodríguez Pérez, Ana Gabriela (2007). El espacio apropiado: territorialización del bar Pluma blanca en Hermosillo, Sonora, asesorada por Nelson Minelo; y la tesis de Parrini Roses, José Rodrigo (2005). Panópticos y laberintos: Subjetivación, deseo y corporalidad en una cárcel de hombres.

En la Maestría en Estudios de la Mujer UAM-Xochimilco de la primera generación 1998-2000 a la séptima generación 2008-2010, de ochenta tesis sustentadas solo dos se dedicaron a los estudios de masculinidades. La primera es de Celmy Teresa, Noh Poot (2007) "Homosexualidades entre varones de Mérida, Yucatán: una sexualidad estigmatizada", asesorada por el Dr. Nelson Minello del Colegio de México; y la de Bolaños Ceballos, Fernando (2008) "La imagen de un fenómeno en movimiento: Un acercamiento a la intervención con hombres que ejercen violencia contra la pareja", asesorada por Dra. Martha Torres Falcón de la UAM-Azcapotzalco.

En la Maestría en Demografía del COLMEX identificamos los trabajos de Colín Paz, Yeimi Alejandra (2005), que presentó la tesis El aborto ¿Un duelo para los varones? Un estudio de casos. Cuyo asesor fue Juan Guillermo Figueroa Perea, y en la Maestría en Estudios de Asia y África la disertación de Escalante Sandoval, Miguel Fernando (2003), Masculinidady representaciones del sexo entre hombres en Japón.

Y por último en CIESAS, Montiel, Oscar (2009), disertó en torno al tema Trata de personas: padrotes, iniciación y modus operandi. Este trabajo es pionero en México y además, obtuvo el premio "Sor Juana Inés de la Cruz" de INMUJERES.

\section{PALABRAS FINALES}

El feminismo ha aportado a la academia e investigación en las instituciones de educación superior un campo de estudios, mediante visibilización del papel desempeñado por las mujeres en la ciencia: sujetos y objeto de estudio; además ha criticado los paradigmas androcéntricos: misoginia y sexismo que permean en el campo de las ciencias. En el campo de las humanidades y ciencias sociales ha propuesto el estudio de las mujeres desde diversas dimensiones históricas, sociales, económicas y culturales, entre otros.

El feminismo trajo al debate teórico el tema del patriarcado: el poder y las relaciones de dominación y subordinación entre las mujeres y los hombres, y con él la crítica a la 
masculinidad tradicional, la que privilegia el papel de los hombres en el mundo de lo público y los excluye de sus afectos a vivir una paternidad amorosa, a reproducir la violencia en sus diversas modalidades, a inhibir el cuidado y atención de la salud sexual y reproductiva, por mencionar algunos de los temas.

Los hombres profeministas - de la Academia Mexicana de los Hombres- han retomado el feminismo histórico -como teoría- para explicar los procesos socioculturales que lo ha construido con reproductores de una masculinidad hegemónica y buscar nuevas formas de relaciones con las mujeres y los hombres. Desde la premisa por transformar el conocimiento y analizar los problemas emergentes, se organizan los primeros seminarios y talleres acerca de los estudios de las masculinidades, en especial en la UNAM, la UAM y el Colegio de México.

Los aportes tanto de los estudios feministas como de las masculinidades se han concretado en un nuevo campo de investigación, de docencia y en especial de profesionales tanto de pregrado como posgrado. Por eso es necesario que en los centros universitarios y de investigación se promueva:

1. Crear cátedras, seminarios y cursos curriculares tanto en las licenciaturas como en los posgrados donde se analicen las diversas posturas teóricas del feminismo y de las masculinidades.

2. Promover y financiar investigaciones interdisciplinarias en cada una de las Facultades, Institutos y Centros de Investigación, por medio de las líneas de investigación para diagnosticar la problemática que enfrentan las mujeres y hombres en el entorno local y regional, y posibilite la elaboración de propuestas incluyentes donde estén presentes las diversas posturas epistémicas feministas y de la masculinidad.

3. Es necesario revisar autocríticamente si los hombres que estudian a otros hombres o las masculinidades están en proceso de cambio o mantienen las resistencias que dictan y reproduce el sistema patriarcal, que tanto cuestiona el feminismo y un sector de los estudios de los varones y las masculinidades.

4. Reconocer los aportes de las feministas en los estudios de los hombres y la masculinidad en cada institución, y visibilizar sus contribuciones teóricas en este campo de estudio.

5. En las y los estudiosos/as del feminismo y la masculinidad debemos evitar la competencia y la invisibilización de la producción científica de los/as otros/as que no coinciden o discrepan con nuestras posturas y acercamientos metodológicos -quién sabe, quién cita a quién, quién investiga y publica más, quién dirige o está en tal o cual grupo o red académica-, pues estas son formas de reproducción de la mercantilización del conocimiento propio del sistema patriarcal que se presenta como los indicadores de "alta producción científica" en el contexto neoliberal de la investigación.

6. Crear un sitio web gratuito interinstitucional que resguarde los resultados de investigación de los estudios feministas y masculinidad con servicio interno y externo para visibilizar la contribución de las y los especialistas en la generación del conocimiento en esta área de estudios. 


\section{BIBLIOGRAFÍA}

Alatorre, Javier (2000): Área de Masculinidad. Seminario. Programa Universitario de Estudios de Género. México, PUEG.

Amuchástegui, Ana (2006): “Masculinidad(es)?: Los riesgos de una categoría en construcción”, en Careaga, Gloria y Cruz, Salvador (coord.): Debates sobre masculinidades. Poder, desarrollo, políticas públicas y ciudadanía, pp. 159-181. México: PUEG.

Amuchástegui, Ana y Szasz, Ivonne (2007): Sucede que me canso de ser hombre: Relatos y Reflexiones Sobre Hombres y Masculinidades en México. México: Colegio de México.

Careaga, Gloria y Cruz, Salvador (2006): Debates sobre masculinidades. Poder, desarrollo, políticas públicas y ciudadanía. México, PUEG.

Cazés, Daniel (1998): "Metodología de género en los estudios de hombres", en La ventana, núm. 8, diciembre, pp. 100-120. México, Universidad de Guadalajara.

Cazés, Daniel (1994): "La dimensión social del género: posibilidades de vida para hombres y mujeres en el patriarcado", en CONAPO: Antología de la sexualidad humana, pp. 335-388. México, D.F.: Consejo Nacional de Población-Porrúa.

Conferencia Mundial de la Mujer (1995): Declaración y Plataforma de Acción de Beijing. La Cuarta Conferencia Mundial sobre la Mujer, 4-15 de septiembre. Beijing, China. [On line]. Disponible en: http://www.un.org/womenwatch/daw/beijing/pdf/BDPfA\%20S.pdf

Escalante, Tania; Lozano, Ignacio (2014): Debates y Reflexiones en torno a las Masculinidades: Analizando caminos hacia la igualdad de género. México: UNAM.

Núñez Noriega, Guillermo. (2004): "Los hombres y el conocimiento. Reflexiones epistemológicas para el estudio de 'los hombres' como sujetos genéricos", en Desacatos, núm. 15, pp. 13-32.

Miqueo, Consuelo; Barral, María José y Magallón, Carmen (Eds.) (2008): Estudios Iberoamericanos en Ciencia, Tecnología y Salud. España: Prensas Universitarias de Zaragoza.

Misael, Oscar (2008): “Debates y aportes en los estudios sobre masculinidades en México”, en Relaciones, Vol. XXIX, núm. 116, pp. 231-253.

Pinilla, David (2014): "Masculinidades emergentes en México: un acercamiento a los activistas por la diversidad sexual, contra la violencia de género y la igualdad". Informe de investigación de estancia académica, México: PUEG.

Ramírez, Juan Carlos y Uribe, Griselda (Coords.) (2008): Masculinidades. El juego de género de los hombres en el que participan las mujeres. México, Plaza y Valdés, Universidad de Guadalajara, Academia Mexicana Jalisciense de Ciencias, Academia Mexicana de Estudios de Género de los Hombres, Fondo de Población de las Naciones Unidas.

Rivera, Cirilo (2014):“De la academia a la sociedad civil”. La manzana. Núm. 11, [On Line]. Disponible en: http://www.estudiosmasculinidades.buap.mx/num13/sociedad.htm 
Rivera Gómez, Elva (2010): De la manifestación al aula. Silencios, saberes e inequidades de género en la Universidad Autónoma de Puebla, 1972-2001. Tesis (Doctorado en Historia y Estudios Regionales), Xalapa, Veracruz. México. Universidad Veracruzana, Instituto de Investigaciones Histórico-Sociales.

Rivera, Elva (2006):"Horizontes para Caminar. Los Estudios de Género, Masculinidad y Universidad". La manzana. Núm. 1, [On Line]. Disponible en: http://www.estudiosmasculinidades.buap. $\mathrm{mx} /$ paginas/frames.htm 